\title{
A Traffic Aware Energy Saving Scheme for Multicarrier HSPA+
}

\author{
Maliha U. Jada ${ }^{1,2}$, Mario García-Lozano ${ }^{2}$, and Jyri Hämäläinen ${ }^{1}$ \\ 1 Department of Communications and Networking, Aalto University, Espoo (Finland) \\ \{maliha.jada, jyri.hamalainen\}@aalto.fi \\ 2 Department of Signal Theory and Communications, BarcelonaTech (UPC), \\ Barcelona (Spain) \\ mariogarcia@tsc.upc.edu
}

\begin{abstract}
In the near future, an increase in cellular network density is expected to be one of the main enablers for the newly introduced challenging capacity goals. This development will lead to an increase in the network energy consumption. In this context, we propose an energy efficient dynamic scheme for HSDPA+ (High Speed Downlink Packet Access-Advanced) systems aggregating several carriers. In the proposed scheme the network adapts dynamically to the network traffic. The scheme evaluates whether node-B deactivation is feasible without compromising the user flow throughput. Furthermore, instead of progressive de-activation of carriers and/or node-B switch-off, we evaluate the approach where feasible combination of inter-site distance and number of carriers is searched to obtain best savings. The solution exploits the fact that re-activation of carriers might permit turning off the BSs earlier at relatively higher load than existing policies, which provides the highest energy saving. Remote electrical downtilt is also considered as a means to reduce the utilization of lower modulation and coding schemes (MCS) in the new extended cells. This approach promises significant energy savings when compared with existing policies - not only for low traffic hours but also for medium load scenarios.
\end{abstract}

Key words: Multicarrier HSPA+, energy saving, cell switch off, carrier management

\section{Introduction}

Due to the increase in demand for mobile broadband services, mobile network vendors are preparing to $1000 \times$ mobile data traffic increase between 2010 and 2020 [1]. This is basically nothing new: According to [2] wireless capacity has already increased more than $10^{6} \times$ since 1957 . Whereas $5 \times$ comes from improvements in modulation and coding schemes (MCS), 1600× increase is due to the reduction in cell sizes. It is widely accepted that the new $1000 \times$ objective comes hand in hand with a further reduction in distances between transmission points. Network densification allows higher spatial reuse and so it allows higher area spectral efficiency $\left[\mathrm{bits} / \mathrm{s} / \mathrm{Hz} / \mathrm{km}^{2}\right]$. On the other hand, considering that base 
stations (BSs) contribute the most to the energy consumption $[3,4,5]$, future hyper-dense network deployments may negatively impact on the operational costs and carbon emissions.

It has become an important goal for industry and academia to reduce the energy consumption of mobile networks over coming years. Energy efficiency is one of the key challenges in the evolution towards beyond fourth generation $(4 \mathrm{G})$ mobile communication systems. Yet, focusing in future systems is not enough since High Speed Packet Access (HSPA) and Long Term Evolution (LTE) will serve and coexist in the next decade, with probably a more tight integration in future releases of the standards [6]. In particular, HSPA is currently deployed in over 500 networks and it is expected to cover $90 \%$ of the world's population by 2019 [7]. So it will serve the majority of subscribers during this decade while LTE continues its expansion in parallel and gain constantly large share of users.

Among the advantages in the latest releases of HSPA (HSPA+), multicarrier utilization is considered as an important performance booster [8] but it has not been extensively studied from the energy efficiency perspective so far. Given this, the focus of our study is in the reduction of energy consumption through dynamic usage of multiple carriers combined with the BS (node-B) switch-off.

Various BSs turn off strategies have been extensively studied as means for energy saving. Since cellular networks are dimensioned to correctly serve the traffic at the busy hour, the idea behind these strategies is to manage the activity of BSs in an energy-efficient manner while simultaneously being able to respond the traffic needs dynamically. Thus, the focus is on strategies where underutilized BSs are switched off during low traffic periods $[3,9,10]$. In order to guarantee coverage, switch-off is usually combined with a certain power increase in the remaining cells, but still providing a net gain in the global energy saving. However, this is not a straight-forward solution from practical perspective: common control channels also require a power increase and electromagnetic exposure limits must be fulfilled [11]. Remote electrical downtilt lacks these problems, it positively impacts the noise rise and received powers, and thus the coverage for common control channels could be expanded without increasing their power [12]. More recently, BS cooperation has also been proposed to cover the newly introduced coverage holes when switch-off is applied [13].

Algorithms that minimize the energy consumption do also have an impact on the system capacity. The work in [14] studies these conflicting objectives and investigates cell switching off as a multiobjective optimization problem. This tradeoff should be carefully addressed, otherwise the applicability of a particular mechanism would be questionable. Yet, not many works consider the capacity issue in detail and many of the contributions just introduce a minimum signal-tonoise plus interference ratio (SINR) threshold, which allows to compute a minimum throughput or outage probability to be guaranteed. Consequently, capacity does not remain constant before and after the switch off. Indeed [15] strongly questions the applicability of cell switch off combined with power increases as a feasible solution for many scenarios. 
Very few works evaluate energy saving gains obtained by advantageous use of the multi-carrier option. The works [16] and [17] respectively deal with HSPA and LTE when two carriers are aggregated and evaluate whether the additional carrier can be de-activated when load decreases and BSs are not powered off.

The current work deals with the reduction of energy consumption in HSPA+ by means of a strategy that combines partial and complete node-B switch off with antenna downtilt. Utilization of multiple carriers is evaluated as an additional degree of freedom that allows more energy effective network layouts. The number of available carriers is dynamically managed in combination with full BS turn off. This last action provides the highest energy saving. For this reason, instead of progressive de-activation of carriers until the eventual node-B turn off, we evaluate the combination (inter-site distance, number of carriers) that gives best energy saving. The solution exploits the fact that activation of previously shutoff carriers might permit turning off the BSs earlier at relatively higher load than existing policies. The new scheme promises significant energy savings when compared with existing policies - not only for low traffic hours but also for medium load scenarios.

The paper is organized in five sections. Section II discusses about the advantages and possibilities of multicarrier HSPA+. Section III describes the system model. In Section IV we discuss about the BS shut-off scheme and Section V is devoted to results and discussion. Conclusions are drawn in Section VI.

\section{Multicarrier HSPA+}

Latest releases of HSPA offer numerous upgrade options with features such as higher order modulation, multi-carrier operation and multiple input multiple output (MIMO). Evolution from initial releases is smooth since MCS update and multicarrier are unexpensive features [18]. These advantages have motivated $65 \%$ of HSPA operators to deploy HSPA+, as recorded December 2013 [7].

HSPA has evolved from a single carrier system to up to 8-carrier aggregation (8C-HSDPA). So, multicarrier operation can be supported in a variety of scenarios depending on the release, indicated in Table 1 for the downlink (HSDPA). Note that the uplink just allows dual cell since release 9. Multicarrier capability being important advantage that affect the system performance $[8,19]$ :

- It scales the user throughput with the number of carriers reaching a top theoretical speed of $672 \mathrm{Mbps}$ on the downlink when combining 8C-HSDPA with $4 \times 4$ MIMO.

- It also improves spectrum utilization and the system capacity because of the load balancing between carriers.

- Multicarrier operation improves the user throughput for a given load at any location in the cell, even at the cell edge, where channel conditions are not good. Note that other techniques such as high order modulation combined with high rate coding or the transmission of parallel streams with MIMO require high SINRs. Furthermore, it is well known that every order of MIMO 
Table 1. Evolution of multicarrier HSDPA

\begin{tabular}{|c|c|c|}
\hline \multicolumn{2}{|c|}{ Release Name } & Aggregation type \\
\hline $\mathrm{R} 8$ & Dual Cell HSDPA & 2 adjacent downlink carriers \\
\hline \multirow{2}{*}{$\mathrm{R} 10$} & \multicolumn{2}{|c|}{ Dual band HSDPA 2 carriers from 2 different bands } \\
\hline & 4C-HSDPA & Up to 4 carriers from one or 2 bands \\
\hline $\mathrm{R} 11$ & 8C-HSDPA & Up to 8 carriers from one or 2 bands \\
\hline
\end{tabular}

just doubles the rate only for users with good channel strength and no line of sight, while on cell edge MIMO just provides diversity or beamforming gain.

Regarding the availability of bandwidth, dual-carrier is currently mainstream solution. 8C-HSDPA is a likely option for scenarios in which bands from second generation $(2 \mathrm{G})$ systems are intensively refarmed or the use of unpaired bands as supplemental downlink is introduced [20]. On the other hand, scalable bandwidth for HSPA would also allow a more gradual refarming process and availability of new bandwidth pieces for aggregation [21]. However, the most interesting option would be a holistic management of the operator's spectrum blocks, with concurrent operation of GSM, HSPA and LTE that would allow an efficient resource sharing among technologies [22]. This multiaccess management can consider both quality of service (QoS) and energy efficiency as described herein.

\section{System Model}

The BS shut-off scheme presents a well-defined solution for a problem of underutilized network elements. However, as previously stated, this action should be performed without compromising the system performance. This section presents the model to assess coverage and capacity dimensioning.

\subsection{Coverage Model}

Let us assume the downlink of an HSPA+ system. At the link level, 30 modulation and coding schemes (MCS) are adaptively assigned by the scheduler based on the channel quality indicator (CQI) reported by user equipments (UEs). Given the channel condition and the available power for the high speed physical downlink shared channel (HS-PDSCH) $P_{\mathrm{HS}-\mathrm{PDSCH}}$, the scheduler selects the MCS that would guarantee a $10 \%$ block error rate (BLER) for each user per transmission time interval (TTI).

The CQI reported on the uplink can be approximated using the SINR $(\gamma)$ at the UE for the required BLER as [23]:

$$
C Q I= \begin{cases}0 & \text { if } \gamma \leq-16 \mathrm{~dB} \\ \left\lfloor\frac{\gamma}{1.02}+16.62\right\rfloor & \text { if }-16 \mathrm{~dB}<\gamma<14 \mathrm{~dB} \\ 30 & \text { if } 14 \mathrm{~dB} \leq \gamma\end{cases}
$$


Throughput of UE $i$ depends on the number of allocated carriers and the SINR at each carrier $f$ :

$$
\gamma_{i}=\frac{\frac{N_{\text {code }} P_{\text {code }}}{L_{s, i}}}{(1-\alpha) \frac{P_{\mathrm{tot}}-P_{\text {code }}}{L_{s, i}}+\sum_{j \neq s}\left(\bar{\rho}_{j} \frac{P_{\mathrm{tot}}}{L_{j, i}}\right)+P_{\mathrm{N}}} 16,
$$

where:

- For the sake of clarity, index referring to carrier $f$ has been omitted.

- $L_{j, i}$ is the net loss in the link budget between cell $j$ and UE $i$ for carrier $f$. Note that index $s$ refers to the serving cell.

- $P_{\text {tot }}$ is the carrier transmission power. Without loss of generality, it is assumed equal in all cells of the scenario.

- Intercell interference is scaled by neighbouring cell load $\bar{\rho}$ at $f$ (carrier activity factor).

- $P_{\mathrm{N}}$ is the noise power.

- $P_{\text {code }}$ is the power allocated per HS-PDSCH code. Note that all codes intended for a certain UE shall be transmitted with equal power [24]. So, considering an allocation of $N_{\text {code }}$ codes and a power $P_{\mathrm{CCH}}$ for the control channels that are present in $f$, then $P_{\text {code }}=\frac{P_{\text {tot }}-P_{\mathrm{CCH}}}{N_{\text {code }}}$.

- The orthogonality factor $\alpha$ models the percentage of interference from other codes in the same orthogonal variable spreading factor (OVSF) tree. Our model assumes classic Rake receivers, in case of advanced devices (Type 2 and Type 3/3i) [25], their ability to partially suppress self-interference and interference from other users would be modelled by properly scaling the interfering power $[26]$.

At the radio planning phase, a cell edge throughput is chosen and the link budget is adjusted so that the corresponding SINR (CQI) is guaranteed with a certain target probability $p_{\mathrm{t}}$. Given that both useful and interfering powers are log-normally distributed, the total interference is computed following the method in [27] for the summation of log-normal distributions. Coverage can be computed for any CQI and so, the boundary in which MCS $k$ would be used with probability $p_{\mathrm{t}}$ can be estimated. This allows finding the area $A_{k}$ in which $k$ is allocated with probability $\geq p_{t}$. Fig. 1 shows an example for a tri-sectorial layout with node-Bs regularly distributed using an inter-site distance (ISD) of $250 \mathrm{~m}$. The shape of the final CQI rings largely depends on the downtilt and antenna pattern at each carrier. The example considers a multiband commercial antenna and optimized downtilt to maximize capacity.

Rings distribution will expand or reduce following the load in other cells. Fig. 2 shows the pdf for CQIs 15 to 30 for 2,4 or 8 carriers and the same cell load, and so different load per carrier $\bar{\rho}(f)$. Interference is spread among the different carriers and so the probability of allocating higher CQIs increases with the number of carriers. This has an impact on cell capacity and so, the next subsection is devoted to describe its model. 


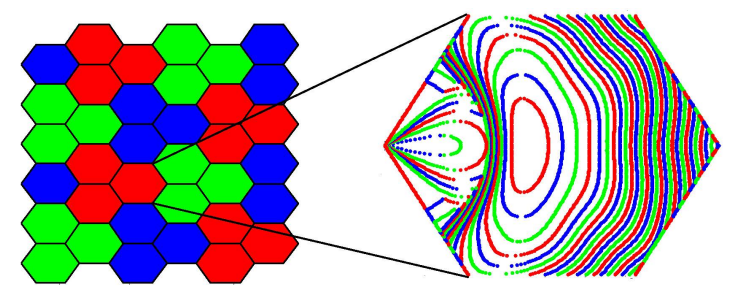

Fig. 1. Probabilistic CQI ring distribution in tri-sectorial regular layout.

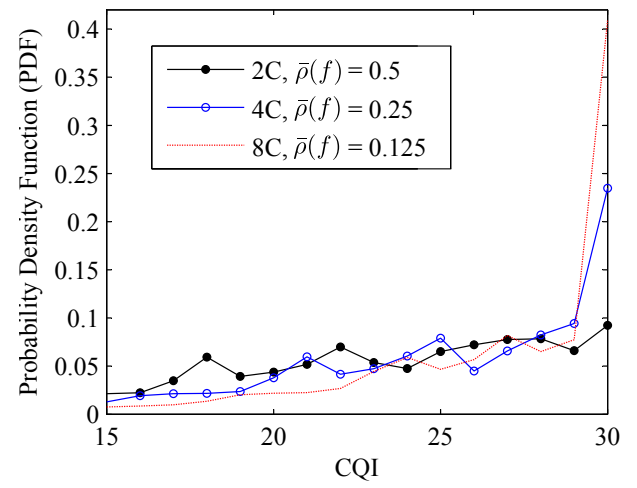

Fig. 2. CQI pdf for 2, 4 and 8 carriers and same cell load.

\subsection{Capacity Model}

The capacity model largely follows [28]. We define cell capacity as the maximum traffic intensity that can be served by the cell without becoming saturated. Note that the cell load is evenly distributed among all carriers, so for the sake of clarity and without loss of generality we will proceed the explanation assuming one single carrier and the index $f$ will be omitted. A round robin scheduler is assumed, more refined options would just shift absolute throughput values.

Cell Capacity Let's assume the traffic to be uniformly distributed in the cell. Data flows arrive according to a Poisson process with rate $\lambda$ per area unit. Flow sizes are independent and identically distributed with average size $\mathrm{E}(\sigma)$. So, the cell load or fraction of time in which the scheduler must be active is:

$$
\bar{\rho}=\lambda A_{\text {cell }} \times \sum_{k=1}^{30} \frac{\mathrm{E}(\sigma)}{c_{k}} p_{k} \leq 1,
$$

where $A_{\text {cell }}$ is the cell area, $c_{k}$ is the code rate associated to MCS $k$, and $p_{k}$ is the probability of using MCS $k, p_{k}=A_{k} / A_{\text {cell }}$. Since the cell load is bounded to one, the maximum throughput that can be served $(\bar{\rho}=1)$, or cell capacity is:

$$
\bar{C}=\left(\sum_{k=1}^{30} \frac{p_{k}}{c_{k}}\right)^{-1} .
$$


At any given load, the observed throughput (served) would be given by $\bar{\rho} \times \bar{C}$.

Flow Throughput Actions to provide energy savings should not compromise the QoS and so the user flow throughput should not been altered. Hence, this has been used as performance metric. Since the scheduler is shared among the users in the cell, serving time depends on the cell load and allocated MCS. So the contribution to the cell load from users at $A_{k}$ (fraction of time that should be allocated by the scheduler) is given by

$$
\bar{\rho}_{k}=\frac{\lambda A_{k} \times \mathrm{E}(\sigma)}{c_{k}} .
$$

It is immediate that $\bar{\rho}=\sum_{k} \bar{\rho}_{k}$. Given that all users in the cell share the same scheduler, by using Little law's the mean flow duration $t_{k}$ for a user in $A_{k}$ can be computed $t_{k}=N_{k} / \lambda A_{k}$ where $N_{k}$ is the average number of users in $A_{k}$. Then the flow throughput $\tau_{k}$ for users being served with MCS $k$ is:

$$
\tau_{k}=\frac{\mathrm{E}(\sigma)}{t_{k}}=\frac{\lambda A_{k} \times \mathrm{E}(\sigma)}{N_{k}},
$$

Considering the underlaying Markov process [28], it can be found the stationary distribution of the number of active users in each $A_{k}$ and its average value, $N_{k}=\frac{\bar{\rho}_{k}}{1-\bar{\rho}}$, which yields:

$$
\tau_{k}=c_{k}(1-\bar{\rho}),
$$

and the average flow throughput at cell level:

$$
\bar{\tau}=\sum_{k=1}^{30} p_{k} c_{k}(1-\bar{\rho}) .
$$

where $\bar{\rho}$ captures the own cell load and $p_{k}$ is affected by the load in neighbouring cells, which modifies SINR values, CQI rings and so $A_{k}$ values $\forall k$.

\section{Node-B Shut off Scheme and Energy Model}

At medium load levels, it is not possible to just shut off BSs without user throughput degradation. On the other hand, at this stage some carriers might have already been de-activated due to underutilization or could even be obtained from other low loaded systems if radio resources are jointly managed. The transition of a network configuration (in terms of ISD and active number of carriers) takes place at a certain load threshold. This load threshold depends upon the possibility of deactivating or reactivating specific number of carriers, and the number of BSs that could remain active or could be shutoff respectively, maintaining the requested average flow throughput. Any shut off is followed by an update of downtilt angles in remaining cells to maximize capacity under the new conditions, and so the use of highest possible MCS. 
After performing node-B shut off, the higher load of the new expanded cells is again evenly distributed over the total frequency resources. This high load includes the user traffic of the switched off node-Bs, which has to be accommodated by the remaining active ones. Considering $v$ as the ratio ISD ${ }^{\text {new }} /$ ISD $^{\text {initial }}$ and that cell area $A_{\text {cell }}$ is proportional to the cell square radius, from (3), the relation between the cell load with new ISD $\bar{\rho}^{\text {new }}$ and with initial ISD $\bar{\rho}^{\text {initial }}$ is given by,

$$
\bar{\rho}^{\text {new }}=v^{2} \times \bar{\rho}^{\text {initial }} .
$$

Although the use of more carriers will account for a certain increase in energy consumption, the saving for switching off some BSs is much higher.

The metric used for the analysis of energy consumption is energy consumed per unit area (E/A). Assuming an entire parallel system at the node-B to handle each carrier, the energy consumed per unit area $\left(\mathrm{kWh} / \mathrm{km}^{2}\right)$ is given as $[4,16,29]$ :

$$
E / A=\frac{N_{\text {site }} \cdot N_{\text {sector }} \cdot N_{\text {car }} \cdot\left[P_{\text {oper }}+\left(\bar{\rho}(f) \cdot P_{\text {in }}\right)\right] \cdot T}{A_{\text {tot }}},
$$

where, $N_{\text {site }}, N_{\text {sector }}, N_{\text {car }}$ are the number of sites, sectors per site and carriers respectively. $P_{\text {oper }}$ is the operational power which is the load independent power needed to operate the node-B. On the other hand, $P_{\text {in }}$ is the power consumed to eventually obtain the required power at the antenna connector. Finally, $T$ is the time duration the particular load remains in the piece of network under study and $A_{\text {tot }}$ is the total evaluated area containing $N_{\text {site }}$ sites. Any change in $N_{\text {site }}$ and/or $N_{\text {car }}$ implies the corresponding update in $\bar{\rho}(f)$.

\section{Results}

In order to quantify the gains that can be achieved by an intelligent joint management of carriers and node-Bs, the system performance is evaluated in terms of average flow throughput $(\bar{\tau})$. Three cases have been evaluated: 2,4 and 8 carriers are initially used to serve an aggregated cell load of 1 . This load is evenly distributed among the carriers, $\bar{\rho}(f)=0.5,0.25$, and 0.125 . Given this, three scenarios are defined considering the $\bar{\tau}$ value to be respected (Table 2).

The reference network has node-Bs regularly deployed and considering an ISD $=250 \mathrm{~m}$. Therefore after a first shut off, the new ISD would be $500 \mathrm{~m}$, and a second implies ISD $=750 \mathrm{~m}$. Other network parameters are provided in Table 3 .

Fig. 3 represents the power consumption per unit area for decreasing cell load values and showing the transition points that should be used to guarantee the target user flow throughput after the network update. In each subplot four cases are represented:

- Initial: Power consumption under the initial network configuration, without changes. It can be seen that it just depends on the system load and so the power consumption is just slightly reduced.

- BSO: Base station shut off. Classic model in which node-Bs are successively shut off whenever the load allows to still keep the target flow throughput. 
Table 2. Evaluated scenarios.

\begin{tabular}{llll}
\hline & Scenario 1 & Scenario 2 & Scenario 3 \\
\hline Targeted $\bar{\tau}$ & $5.76 \mathrm{Mbps}$ & $21.80 \mathrm{Mbps}$ & $60.53 \mathrm{Mbps}$ \\
\hline
\end{tabular}

Table 3. Network Parameters

\begin{tabular}{ll}
\hline Parameter & Value \\
Operating bands & $2100 \mathrm{MHz}, 900 \mathrm{MHz}$ \\
Inter-Site distances & $250 \mathrm{~m}, 500 \mathrm{~m}, 750 \mathrm{~m}$ \\
Number of sites, for each ISD & $108,27,12$ \\
Optimum downtilt angles, for each ISD $19.5^{\circ}, 12.5^{\circ}, 11.5^{\circ}$ \\
Macro BS transmission power & $20 \mathrm{~W}$ \\
Transmission power per user & $17 \mathrm{~W}$ \\
Control overhead & $15 \%$ \\
BS antenna gain & $18 \mathrm{~dB}$ \\
Body loss & $2 \mathrm{~dB}$ \\
Cable and connection loss & $4 \mathrm{~dB}$ \\
Noise power & $-100.13 \mathrm{dBm}$ \\
Propagation model & Okumura-Hata \\
Shadow fading std. deviation & $8 \mathrm{~dB}$ \\
Cell edge coverage probability & 0.99 \\
\hline
\end{tabular}

- CSO: Carrier shut off. Generalization of the DC-HSDPA case in [16], carriers are progressively shut off with load reduction.

- JM: Joint management. The proposal of the current work. Power off of BSs and carriers are jointly managed and re-activation of carriers is a valid option if that justifies earlier full BSs shut off and so a net energy saving.

Each tag in the plot shows the transition points in terms of (ISD, number of active carriers). Since the load is progressively reduced, the pictures should be read from right to left. For example, for the BSO case in Scenario 1, the transition points evolve as $(250,2) \rightarrow(500,2) \rightarrow(750,2)$, note how the last case can only be implemented for cell loads of $10 \%$, meaning a $5 \%$ of load per carrier.

The joint management allows earlier BS shut off and transition points fall below the other options, thus having clearly less power consumption without performance degradation. It can be seen how JM allows using ISD $=750 \mathrm{~m}$ as soon as the cell load falls below 0.8. For Scenario 2, ISD can be increased from 250 to 500 for high loads, and $750 \mathrm{~m}$ can be used once the load falls below 0.5. Scenario 3 is the most restrictive since it starts with the maximum possible carriers at the current HSPA + standard. So there is less flexibility with respect to the other cases and the savings are just slightly better. For illustrative purposes, it has been included the off-standard case in which up to 10 carriers are used, it can be seen how energy savings are again important. This way, multiaccess 


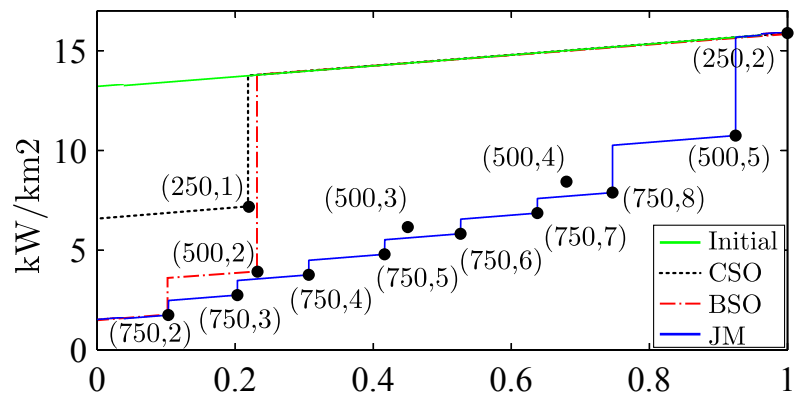

Cell load of initial network with $\mathrm{ISD}=250 \mathrm{~m}$

(a) Scenario 1

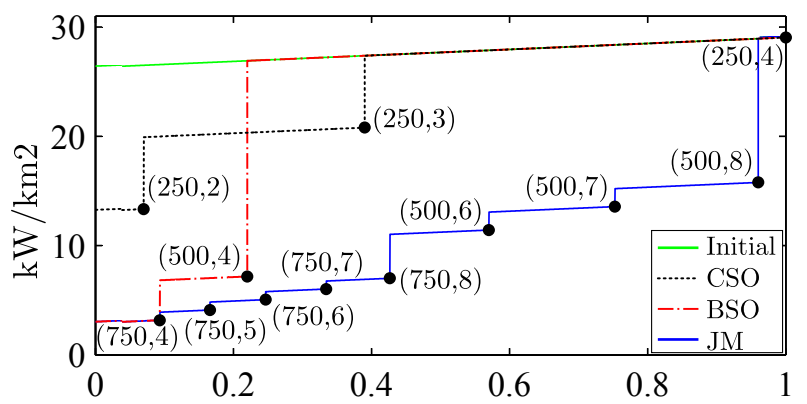

Cell load of initial network with $\mathrm{ISD}=250 \mathrm{~m}$

(b) Scenario 2

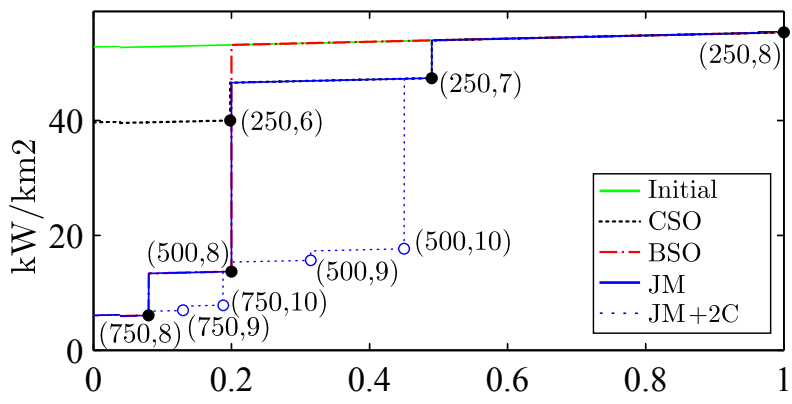

Cell load of initial network with $\mathrm{ISD}=250 \mathrm{~m}$

(c) Scenario 3

Fig. 3. Power consumption per unit area for decreasing cell load values. Transition points indicate the pair (ISD, number of carriers) to be used.

energy saving mechanisms that manage the pool of resources among several systems would make the most of each system load variations.

It is important to note that the horizontal axis represents the equivalent cell load that would be obtained if the network remained unchanged. But obviously, after carrier and/or node-B switch off, the cell load changes. For example, ini- 


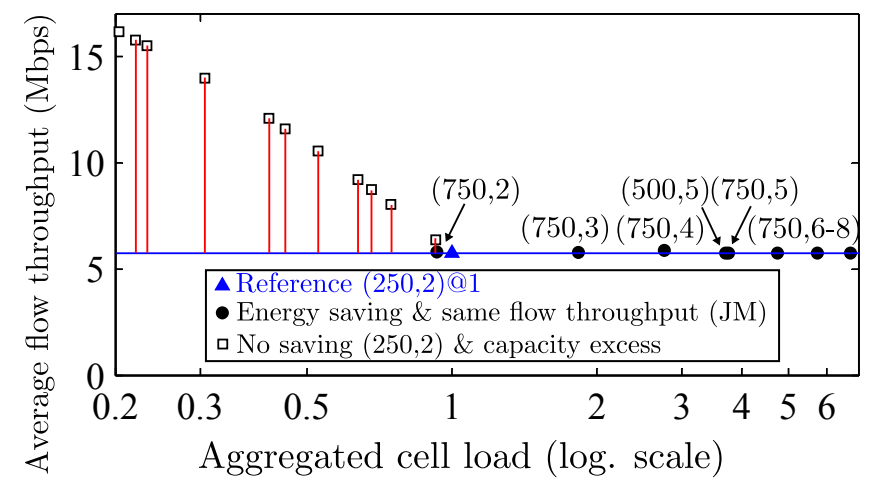

Fig. 4. Transition of cell configuration from initial network setup (scenario 1) to new setups at specific load values and maintaining the QoS requirements (5.75 Mbps).

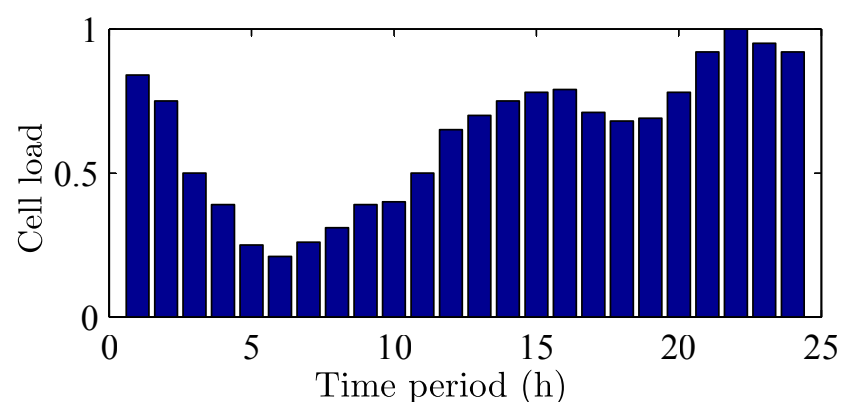

Fig. 5. HSDPA traffic profile over $24 \mathrm{~h}[16]$.

tially the load is 1 ( 0.5 per carrier) and it is not until it is reduced to 0.92 that important energy savings are possible, so we transition from $(250,2) @ 0.92$ to $(500,5) @ 3.7$, recall that since the load per carrier is bounded to 1 , the final aggregated cell value can be $>1$. Besides, it is clear that the cell load increases due to its expansion and the new users to be served, but the QoS is respected, since both $(250,2) @ 1$ and $(500,5) @ 3.7$ provide the same flow throughput.

In order to illustrate how load evolves with every change, Fig. 4 represents the average flow throughput as a function of the aggregated cell load for each configuration proposed by JM (solid symbols). Note the logarithmic scale in the horizontal axis to improve readability. Following Fig. 3a, their evolution is as follows: $(250,2) @ 1 \rightarrow(500,5) @ 3.7 \rightarrow(750,8) @ 6.72 \rightarrow(750,7) @ 5.74$ and so on. If no energy savings mechanisms are implemented, in other words if we remain with the dense node-B deployment, an excess in capacity would be obtained due to load decrement. These situations are represented by empty symbols.

Given the previous results, in the following we consider a realistic profile of daily HSDPA traffic (load) [16] (Fig. 5) and evaluate energy consumption and corresponding savings along time. 

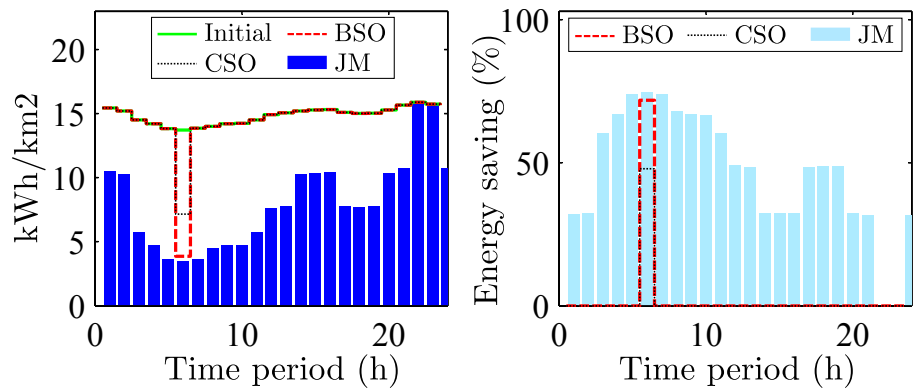

(a) Scenario 1
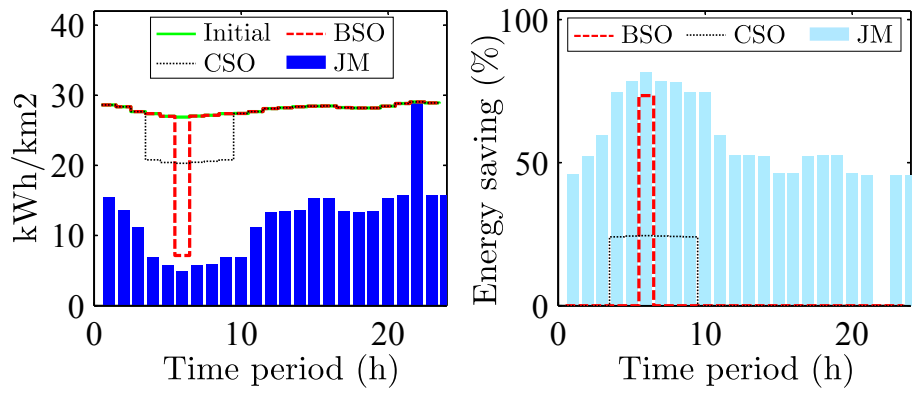

(b) Scenario 2

Fig. 6. Comparison between energy savings (\%) of BSO, CSO and JM.

Fig. 6 represents results for scenarios 1 and 2. In case of Scenario 1, the total energy saving percentage is $45.4 \%$ with $\mathrm{JM}$, whereas it is just $2.8 \%$ with BSO and $1.8 \%$ with CSO. For Scenario 2, gains increase up to $55.8 \%$ for JM, and $2.9 \%, 5.9 \%$ for BSO and CSO respectively. Scenario 3 had an equal saving of just $3.5 \%$ in CSO and JM, with no possible gain with BSO. As previously mentioned this is because scenario 3 is very restrictive and requires a flow throughput of $60.53 \mathrm{Mbps}$. In the hypothetical off-standard case with up to 10 available carriers, energy savings with JM would reach 19.9\%. From Fig. 6 it is also noticeable how small reductions in the load can lead to important savings as it happens with cell load values around $60 \%$. So we can conclude that even at mid-high values, interesting savings are possible when applying the JM approach.

\section{Conclusions}

In this paper we investigated the potential energy savings by shutting off the BSs through the dynamic use of multiple carriers in HSDPA. We have proposed an energy saving scheme in which fewer or additional carriers have been used depending upon the network traffic variations. This is combined with remote electrical downtilts to partially cope with the use of a higher number of lower MCSs. Instead of just guaranteeing a power threshold at the cell edge, or an 
outage probability threshold for data traffic, it is more interesting to ensure that QoS remains unchanged whenever a node-B and/or carrier is shut-off, for this reason the study considers user flow throughput as the performance metric to be respected, which is closely affected by load variations due to cell expansions. Comparison to schemes that progressively shut off network elements (BSO and CSO) has been done, showing clear energy savings with the JM approach.

The main challenge to make the adaptation efficient and flexible is that load fluctuations should be correctly followed. Reiterative traffic patterns can be assessed along time but abnormal temporal or spatial variations could be included in the system by means of a pattern recognition system, e.g. a fuzzy logic based system or a neural network. Further efforts are required in this direction. Moreover, the time for carrier and BS reactivation has not been taken into account for this case study. This will be considered in the future work.

\section{Acknowledgments}

This work was prepared in EWINE-S project framework and supported in part by Finnish Funding Agency for Technology and Innovation (Tekes), European Communications Engineering (ECE) and Efore oyj. The work by Mario GarcíaLozano is funded by the Spanish National Science Council through the project TEC2011-27723-C02-01 (ERDF).

\section{References}

1. Andrew, R.: 2020: The Ubiquitous Heterogeneous Network - Beyond 4G. ITU Kaleidoscope, NSN, Cape Town (2011). [Online]. http://www.itu.int/dms_pub/ itu-t/oth/29/05/T29050000130001PDFE.pdf

2. Chandrasekhar, V., Andrews, J., and Gatherer, A.: Femtocell Networks: a Survey. In: IEEE Comm. Mag., vol. 46, no. 9, pp. 59-67, 2008.

3. Marsan, M., Chiaraviglio, L., Ciullo, D., and Meo, M.: Optimal Energy Savings in Cellular Access Networks. In: IEEE Int. Conf. on Comm. Workshops (ICC Workshops), pp. 1-5, Dresden (2009).

4. Jada, M., Hossain, M.M.A., Hämäläinen, J., and Jäntti, R.: Impact of Femtocells to the WCDMA Network Energy Efficiency. In: 3rd IEEE Broadband Network and Multimedia Technology (IC-BNMT), pp. 305-310, Beijing (2010).

5. Jada, M., Hossain, M.M.A., Hämäläinen, J., and Jäntti, R.: Power efficiency model for mobile access network. In: 21st IEEE Personal, Indoor and Mobile Radio Communications Workshops (PIMRC Workshops), pp. 317-322, Istanbul (2010).

6. Yang, R., Chang, Y., et al.: Hybrid Multi-Radio Transmission Diversity Scheme to Improve Wireless TCP Performance in an Integrated LTE and HSDPA Networks. In: 77th IEEE Vehicular Tech. Conf. (VTC Spring), pp. 1-5, Dresden (2013).

7. 4G Americas: White Paper on 4G Mobile Broadband Evolution: 3GPP Release 11 \& Release 12 and Beyond. Technical Report, 2014.

8. Johansson, K., Bergman, J., et al.: Multi-Carrier HSPA Evolution. In: 69th IEEE Vehicular Tech. Conf. (VTC Spring), Barcelona (2009). 
9. Gong, J., Zhou, S., Niu, Z., and Yang, P.: Traffic-aware Base Station Sleeping in Dense Cellular Networks. In: 18th Int. Workshop on Quality of Service (IWQoS), pp. 1-2, Beijing (2010).

10. Niu, Z.: TANGO: Traffic-aware Network Planning and Green Operation. In: IEEE Wireless Comm., vol. 18, no. 5, pp. 25-29, 2011.

11. Chiaraviglio, L., Ciullo, D., et al.: Energy-efficient Management of UMTS Access Networks. In: 21st Int. Teletraffic Congress (ITC), pp. 1-8, Paris (2009).

12. Garcia-Lozano, M. and Ruiz, S.: Effects of Downtilting on RRM Parameters. In: 15th IEEE Int. Symp. on Personal, Indoor and Mobile Radio Comm (PIMRC), vol. 3, pp. 2166-2170, Barcelona (2004).

13. Han, F., et al.: Energy-efficient Cellular Network Operation Via Base Station Cooperation. In: IEEE Int. Conf. on Communications (ICC), pp. 4374-4378, Ottawa (2012).

14. González G, D., Yanikomeroglu, H., Garcia-Lozano, M., and Ruiz, S.: A Novel Multiobjective Framework for Cell Switch-off in Dense Cellular Networks. In: IEEE Int. Conf. on Comm. (ICC), pp. 2647-2653, Sydney (2014).

15. Wang, X., Krishnamurthy, P., and Tipper, D.: Cell Sleeping for Energy Efficiency in Cellular Networks: Is It Viable?. In: IEEE Wireless Comm. and Networking Conf. (WCNC), pp. 2509-2514, Paris (2012).

16. Micallef, G., Mogensen, P., et al.: Dual-Cell HSDPA for Network Energy Saving. In: 71st IEEE Vehicular Tech. Conf. (VTC Spring), pp: 1-5, Taipei (2010).

17. Chung, Y.-L.: Novel Energy-efficient Transmissions in 4G Downlink Networks. In: 3rd Int. Conf. on Innovative Comp. Tech. (INTECH), pp. 296-300, London (2013).

18. Borkowski, J., Husikyan, L., and Husikyan, H.: HSPA evolution with CAPEX considerations. In: 8th Int. Symp. on Comm. Systems, Networks Digital Signal Processing (CSNDSP), Poznan (2012).

19. Bonald, T., Elayoubi, S.E., et al.: Radio Capacity Improvement with HSPA+ DualCell. In: IEEE Int. Conf. on Communications (ICC), Kyoto (2011).

20. — : RP-140092 - Revised Work Item: L-band for Supplemental Downlink in E-UTRA and UTRA. 3GPP, Tech. Rep., 2014. [Online]. http://www.3gpp.org/

21. — : TR 25.701 v12.1.0 (Release 12) - Study on scalable UMTS Frequency Division Duplex (FDD) Bandwidth. 3GPP, Tech. Rep., 2014. [Online]. http://www.3gpp.org/

22. NSN: Answering the Network Energy Challenge (whitepaper). Tech. Rep., 2014.

23. Brouwer, F., de Bruin, I., et al.: Usage of link-level performance indicators for HSDPA network-level simulations in E-UMTS. In: Int. Symp. on Spread Spectrum Techniques and Applications (ISSSTA), pp. 844-848, Sydney (2004).

24. — : TR 25.214 v11.8.0 (Release 11) - Physical layer procedures (FDD). 3GPP, Tech. Specification, 2014. [Online]. http://www.3gpp.org/

25. — - TR 25.101 v12.3.0 (Release 12) - User Equipment (UE) Radio Transmission and Reception (FDD). 3GPP, Tech. Rep., 2014. [Online]. http://www.3gpp.org/

26. Rupp, M., Caban, S., et al.: Evaluation of HSDPA and LTE: From Testbed Measurements to System Level Performance. Wiley, Dec. 2011.

27. Beeke, K.: Spectrum Planning - Analysis of Methods for the Summation of LogNormal Distributions. EBU Technical Review, no. 9, 2007.

28. Bonald, T. and Proutière, A., Wireless Downlink Data Channels: User Performance and Cell Dimensioning. In: Annual Int. Conf. on Mobile Comp. and Networking (MOBICOM), San Diego, CA (2003).

29. Arnold, O., Richter, F., Fettweis, G., and Blume, O.: Power Consumption Modeling of different Base Station Types in Heterogeneous Cellular Networks. In: Future Network and Mobile Summit, pp. 1-8, Florence (2010). 